\title{
Favorable Functional Recovery and Stem Stability after Hip Arthroplasty with a Short Metaphyseal Stem in Elderly Patients with Osteoporotic Femoral Neck Fractures
}

\author{
Soong Joon Lee, MD, Kang Sup Yoon, MD, PhD \\ Department of Orthopedic Surgery, SMG-SNU Boramae Medical Center, \\ Seoul National University College of Medicine, Seoul, Korea
}

Purpose: Short stems have recently become widely used; however, concerns about the initial secure fixation of a short stem in osteoporotic bone remain. The aim of this study was to evaluate the short-term clinical and radiological results of using a short cementless metaphyseal stabilizing tapered stem for senile osteoporotic femoral neck fractures.

Materials and Methods: Thirty-eight arthroplasties (31 bipolar hemiarthroplasties and 7 total hip arthroplasties) were performed for osteoporotic femoral neck fractures in patients older than 65 years (10 males and 28 females). The mean age was 76.1 years and the mean follow-up was 2.9 years. We retrospectively evaluated clinical results, focusing on walking performance, thigh pain, and radiologic results, with special regard to signs of stem stability and osteointegration.

Results: Mean Harris hip score was 84.3 points and $68.4 \%$ of patients regained their preoperative walking performance. No patients complained about thigh pain. No osteolysis or loosening was observed during the follow-up, and all but 1 stem showed signs of stable bone ingrowth.

Conclusion: Short, metaphyseal stabilizing tapered stems could be a reliable treatment option for osteoporotic femoral neck fractures.

Key Words: Osteoporosis, Femoral neck fractures, Arthroplasty, Hip replacement

Submitted: November 15, 2018 1st revision: January 6, 2019

Final acceptance: January 28, 2019

Address reprint request to

Kang Sup Yoon, MD

(https://orcid.org/0000-0001-5917-1881)

Department of Orthopedic Surgery, SMG-SNU Boramae Medical

Center, 20 Boramae-ro 5-gil, Dongjak-gu, Seoul 07061, Korea

TEL: +82-2-870-2311 FAX: +82-2-831-2826

E-mail: ksyoondasnu.ac.kr

This is an Open Access article distributed under the terms of the Creative Commons Attribution Non-Commercial License (http://creativecommons. org/licenses/by-nc/4.0) which permits unrestricted non-commercial use, distribution, and reproduction in any medium, provided the original work is properly cited

\section{INTRODUCTION}

Previously, cemented stems were widely used for femoral neck fractures. However, there is a concern about a cement-related hemodynamic complication, which might be fatal for elderly patients with these fractures ${ }^{1}$. Because of the possibility of unexpected embolic events during insertion of cemented stems and shorter operation time with cementless implants, a cementless stem could be a reliable treatment option for femoral neck fractures ${ }^{2,3}$. The use of cementless stems for femoral neck fractures has remarkably increased in the last decade ${ }^{2,3}$. Previously, full-coated tapered stems 
or conventional proximal tapered stems showed favorable results for femoral neck fractures ${ }^{4-7}$. However, concerns remain about the use of conventional cementless stems because of thigh pain due to diaphyseal fixation and risk of unexpected bone loss due to the morphology of the stem ${ }^{8,9)}$.

Recently, short stems have been introduced, and their use in arthroplasty has increased ${ }^{10}$. A short metaphyseal stabilizing stem is a type of a proximal tapered-wedge stem $^{11)}$ with a shorter uncoated portion compared to conventional tapered-wedge stem ${ }^{10}$. The optimal design of a short metaphyseal stabilizing stem would preserve more native bone, and its short length might potentially decrease thigh pain, compared to a conventional proximal tapered stem or full-coated tapered stem ${ }^{11}$. However, the short length of the stem and a relatively small area of bone ingrowth raise concerns about secure fixation, especially in osteoporotic bone. To our knowledge, there has been no study on the results of the use of short metaphyseal stabilizing tapered stems for osteoporotic femoral neck fractures. The aim of this study was to evaluate the short-term clinical and radiological results of the use such stems for osteoporotic femoral neck fractures in patients aged over 65 years. We retrospectively evaluated clinical symptoms including the presence of pain, perioperative changes in walking performance, and the Harris hip score (HHS) at final follow-up, and examined the radiographic results for the presence of osteolysis or loosening and signs of osteointegration. We also evaluated the occurrence of complications after the operation.

\section{MATERIALS AND METHODS}

From 2012 to 2013, 60 displaced femoral neck fractures with osteoporosis which was confirmed by dual-energy X-ray absorptiometry (DEXA) evaluation were treated with arthroplasty with short cementless metaphyseal stabilizing stems (Trilock-BPS stem; DePuy, Warsaw, IN, USA). During the study period, cemented stem was inserted in patients with pathologic fracture, or with life expectancy less than a year. For the patients with coxa valga, another type of cementless stem (ML taper; Zimmer, Warsaw, IN, USA) was inserted. Except for these patients, arthroplasty with short tapered stem was performed for all patients with femoral neck fractures. Fig. 1 shows the flow chart of patients with exclusion and inclusion criteria. During the study period, 6 patients died at our hospital before the 2year follow-up, and 16 patients were not followed-up for 2 years. Of the 16 patients with lost follow-up, 6 patients died outside of our hospital before the 2-year follow-up, 4 patients were refused to visit the clinic because their residence was far from the clinic, and 6 patients were lost to contact. Eventually, 38 arthroplasties in 38 patients with femoral neck fractures treated with Trilock-BPS stems were included in the present study; the minimum follow-up was 2 years (mean, $2.9 \pm 0.8$ years). After approval by the institutional review board of our institution, the patients' medical records and radiologic data was retrospectively evaluated.

There were 10 male and 28 female patients. Mean age was $76.1 \pm 6.4$ years (range, 65-92 years). During the

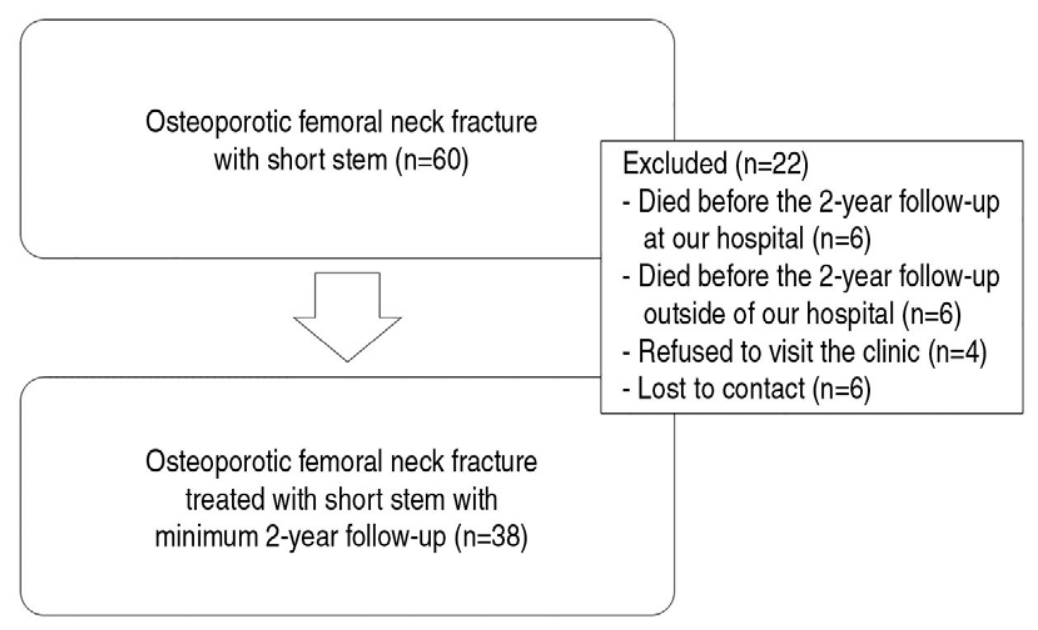

Fig. 1. Flowchart of the patients with inclusion and exclusion criteria. 
study period, 31 bipolar hemiarthroplasties (BHA) and 7 total hip arthroplasties (THA) were performed. All femoral neck fractures were caused by low-energy trauma such as a fall from a height. No patients had a history of major trauma such as a fall from a great height or major traffic accident. In preoperative radiographs, there were 18 hips with Garden type III fractures and 20 hips with Garden type IV fractures ${ }^{12}$. The preoperative proximal femoral geometry was classified as Dorr type C in 12 hips and Dorr type B in 26 hips $^{13)}$. The preoperative walking performance was evaluated by using the Koval grade ${ }^{14)}$. There were 27 patients with grade I, 7 with grade II, one with grade IV, one with grade $\mathrm{V}$, and 2 with grade VI.

Osteoporosis was diagnosed by the DEXA T-score (Lunar Prodigy Advance; GE Healthcare, Madison, WI, USA). In 32 cases, the T-score of the proximal femur was below -2.5. In 4 cases, the T-score of the proximal femur was not available due to fracture of the contralateral hip. In the remaining 2 cases, the T-score of the proximal femur was higher than -2.5 . However, the T-score of the lumbar spine in the latter 6 cases was below -2.5 and met the criteria of osteoporosis. The mean T-score was $-3.07 \pm 1.17$ at the lumbar spine, $-2.98 \pm 0.73$ at the femoral neck, $-2.32 \pm 0.93$ in the trochanteric area, and $-2.78 \pm 0.86$ at the total femur.

All operations were performed in the lateral position. BHA was performed with a modified Gibson posterolateral approach. After femoral neck resection, femoral canal preparation was performed with broaches of increasing sizes. Intimate mediolateral contact was made between the final and the largest available broach and the cortical bone. The contact was confirmed by visual checking of the contact between the medial femoral cortex and medial area of the stem without the presence of remnant cancellous bone. Also, we checked the rotational stability by forceful rotation of the handle of the broach. Then, a trial femoral neck component and a femoral head were implanted, and the reduction was performed. After reduction, the range of motion and stability of the prosthetic joint were evaluated. After removal of the trial component and the broach, the real stem was inserted with a metal head and bipolar cup. Mean operation time was 77.1 minutes from skin incision to closure of the wound. For THA, a modified Hardinge direct lateral approach was performed. First, acetabular preparation was performed, and a cementless cup (Pinnacle cup; DePuy) was inserted. After cup insertion, femoral preparation was performed as for BHA. Then, a trial femoral neck segment and femoral head were implanted, and reduction was performed. After reduction, range of motion and stability were evaluated. Then, the real stem was inserted with a ceramic head (Biolox Delta; CeramTec, Plochingen, Germany). Mean operation time was 94.3 minutes in cases. The overall mean operation time for BHA and THA was 80.3 minutes.

Postoperatively, we evaluated the clinical results on the basis of the HHS, thigh pain, postoperative walking performance, implant survival, and occurrence of complication $\mathrm{s}^{14)}$. On radiographs, the stem insertion angle was evaluated as the angle between the axis of the femur and that of the stem, and hips with a stem insertion angle $>3^{\circ}$ were classified as outliers. According to the stem insertion angle and outlier, stem position was defined as neutral, valgus and varus. In the postoperative X-ray, we evaluated the presence of the distal fixation of the stem by measuring the canal fill index (CFI) with the ratio between the width of the stem and that of the femoral canal at the level of the mid portion of the uncoated area of the stem. When the CFI at the uncoated area was more than $95 \%$, we considered the stem fixation as the potential distal fixation. The presence of osteolysis, loosening or subsidence of the stem was assessed ${ }^{15-17)}$. The signs of bone ingrowth and reactive line around the porous-coated area of the femoral stem, and the signs of stress shielding were also assessed $^{18)}$.

\section{RESULTS}

During the follow-up, no stem was revised for any reason. Mean HHS was $84.3 \pm 14.2$ at the last follow-up. Ten patients with HHS below 70 had radiating pain around the pelvic area due to a spinal problem or general weakness due to poor walking performance. No patients complained of thigh pain. Postoperatively, 26 patients (68.4\%) regained preoperative walking performance. Fig. 2 shows the preoperative and postoperative walking status of the patients.

During operation, 33 stems were inserted in a neutral position, and 5 stems were inserted in a valgus position. No stems were inserted in a varus position. Two intraoperative femoral fractures occurred and treated with wiring. Postoperatively, all stems were securely fixed without signs of loosening or osteolysis until the 


\section{Hip \& Pelvis}

Hip Pelvis 31(1): 11-17, 2019

last follow-up (Fig. 3). There was no implant migration or subsidence. In postoperative X-ray, 8 hips showed CFI $>95 \%$ at the middle of the uncoated area of the stem and showed the sign of the potential distal fixation (Fig. 4). No reactive line of more than $2 \mathrm{~mm}$ was observed. Reactive lines of less than $2 \mathrm{~mm}$ were seen in 16 cases around the tip of the stem. Except for one hip, all stems showed signs of stable fixation with bone ingrowth and without a reactive line around the proximal area of the porous coated surface of the stem. In one case, a reactive line of less than $2 \mathrm{~mm}$ was observed around the greater trochanteric area (Fig. 4). There were no signs of
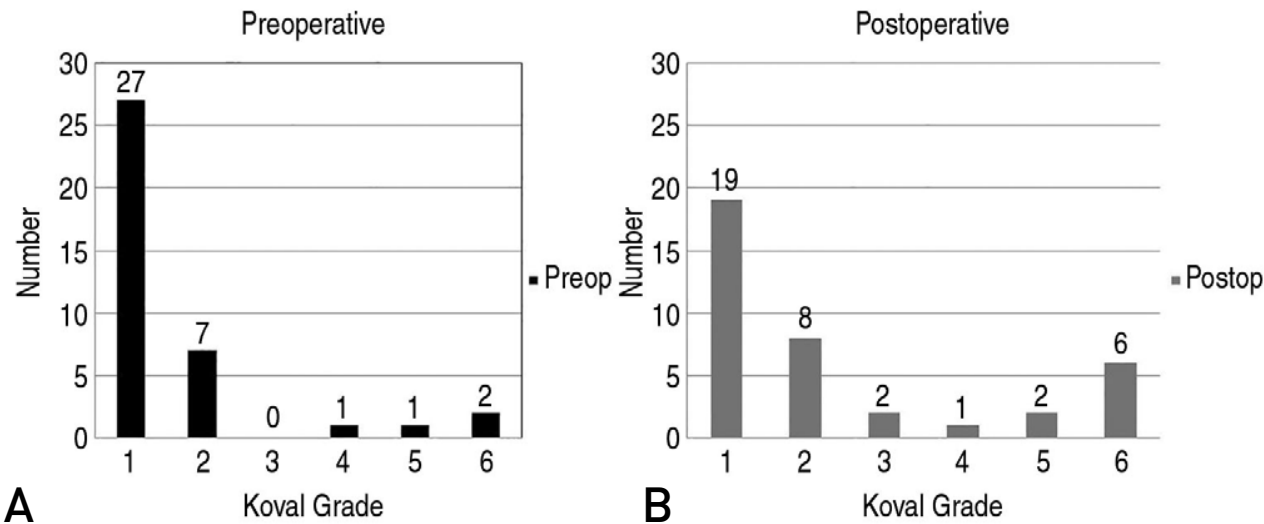

Fig. 2. Preoperative (A) and postoperative (B) walking performance according to the Koval grade.
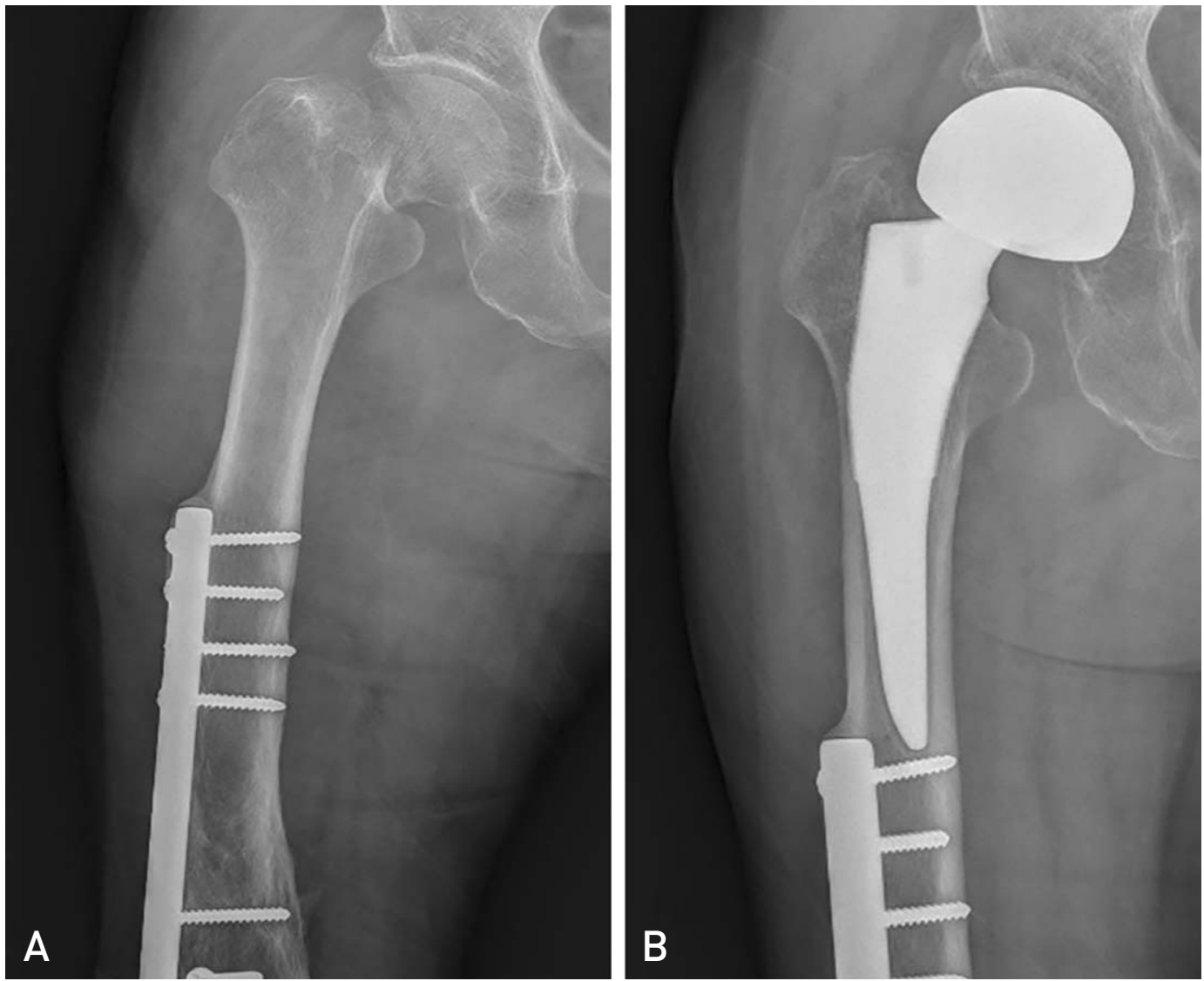

Fig. 3. (A) Preoperative X-ray of an 80-year-old female patient with a history of femoral shaft fracture treated with plate fixation shows a displaced femoral neck fracture. (B) Postoperative 2.5-year X-ray shows stable fixation of the femoral stem without removal of the plate or screw. 


\section{Hip \& Pelvis}

Soong Joon Lee et al. Short Stem for Osteoporotic Femoral Neck Fracture

stress shielding below the lesser trochanteric area.

As to complications, one periprosthetic femoral fracture occurred after a fall from a height and was treated with internal fixation with a plate and wire. During the operation, the stem showed stable fixation and was retained. Radiologic signs of fracture union were achieved at 3 months after the operation. There was no other complication such as infection or dislocation.

\section{DISCUSSION}

The present study showed the favorable short-term functional results of arthroplasty with a short cementless proximal wedged stem for femoral neck fractures with osteoporosis. No thigh pain occurred. No loosening or osteolysis was observed, and bone ingrowth was stable in most cases, even with the short length of the stem. Strength of the study is that we only included patients with osteoporosis diagnosed by DEXA evaluation. Despite the limited area of bone ingrowth, short metaphyseal stems showed excellent clinical and radiological results. Also, the current study showed favorable recovery of walking performance. We believe that favorable functional results with the absence of thigh pain and excellent implant stability might have contributed to the favorable walking performance recovery after hip arthroplasty with shortened stems for osteoporotic femoral neck fractures.

The use of short stems has been increasing in recent years $^{10)}$. A short stem could preserve more native bone and reduce the occurrence of thigh pain ${ }^{11)}$. Short stems have a different philosophy of design and fixation at different area in the proximal femur ${ }^{10}$. Recently, classification of short stems by fixation principle and location of proximal loading has been suggested ${ }^{10)}$. Type I are femoral neck only stems and have only femoral neck fixation ${ }^{10)}$. Type II are calcar loading stems, with fixation in the calcar and the lateral proximal cortex ${ }^{10)}$. Type III are lateral flare calcar loading stem with a tapered trapezoidal geometry and lateral flare, with fixation in the calcar and lateral cortex ${ }^{10)}$. Type IV are shortened tapered conventional stems with a shorter length and reduced distal end in comparison with conventional proximal tapered stems ${ }^{10)}$. The Trilock-BPS stems, which were used in the present study, are type IV stems; they represent a shortened version of the Trilock stem, with the distal
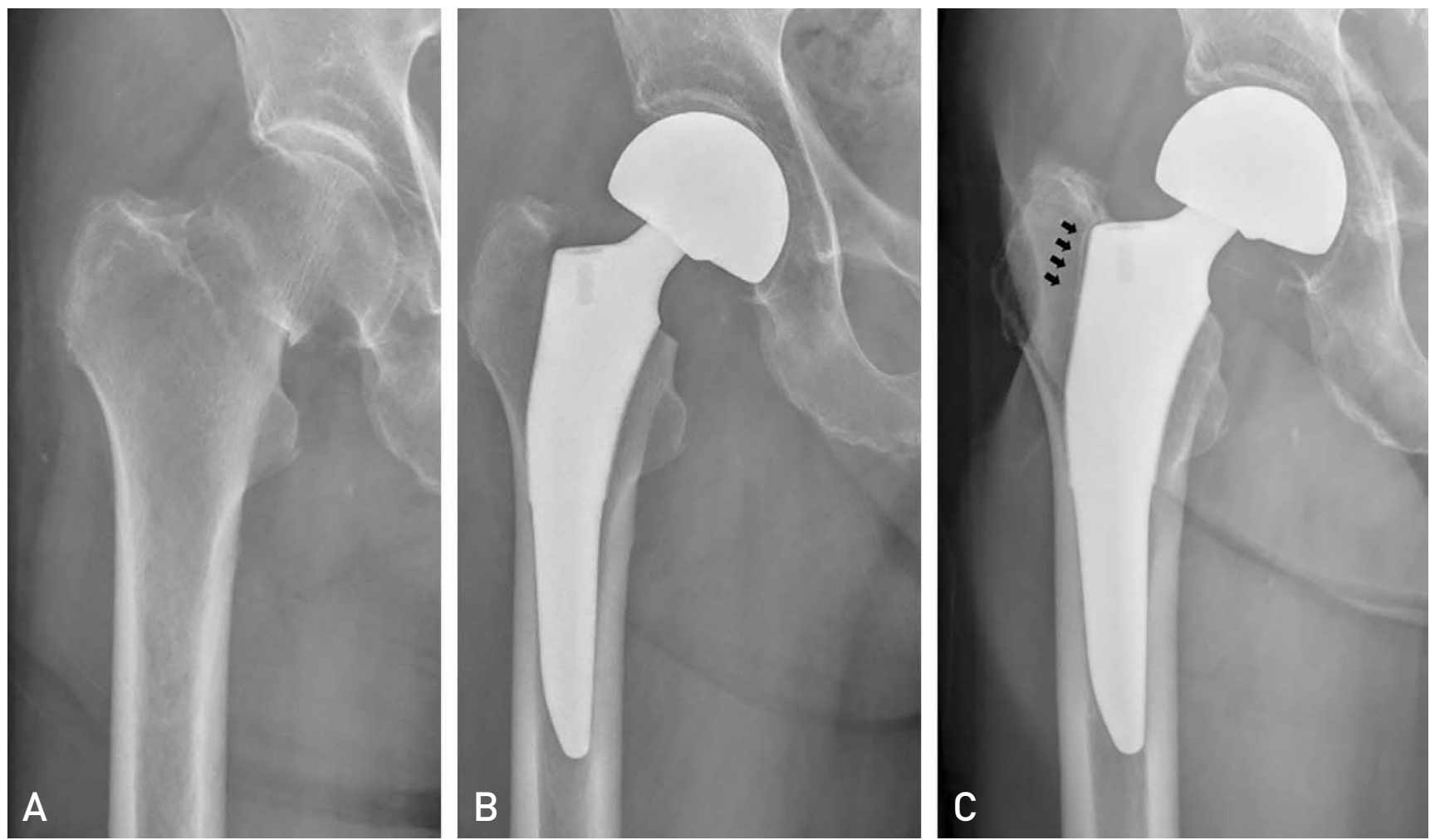

Fig. 4. (A) Preoperative X-ray of a 76-year-old female patient shows a displaced femoral neck fracture. (B) Immediate postoperative X-ray shows distal engaging of the stem. (C) Postoperative 3.6-year X-ray shows a radiolucent line around the greater trochanter (black arrows); however, she had no complaints about pain or discomfort. 
length shortened and the lateral tip curved and contoured for easy insertion and reduction of the disruption of the greater trochanter. Despite their short length, a recent clinical study showed the excellent results of the use of Trilock-BPS stems with mid-term follow-up ${ }^{11}$. However, Dorr $\mathrm{C}$ femurs were excluded from the study ${ }^{11}$. The results of arthroplasty with short stems for osteoporotic femurs have not been well established. The present study showed favorable results of short metaphyseal stabilizing stems for osteoporotic femurs.

Previous studies showed favorable results of conventional cementless stems for femoral neck fractures ${ }^{4-7}$. Proximal wedged stems also showed favorable results for osteoporotic bone without signs of early subsidence ${ }^{19}$. For implantation of a proximal wedged stem, the native proximal femoral anatomy and the shape of the proximal porous coated area of the femoral stem should be matched to ensure bone ingrowth on the porous coated area $^{8}$. In cases of morphological mismatch between native bone and the shape of the stem, distal engagement of the stem may occur, resulting in a failure of bone ingrowth on the proximal porous coated area $^{8}$. In the current study, 8 hips showed the potential distal fixation of the stem, and in one of 8 patients, a radiolucent line of more than $2 \mathrm{~mm}$ was observed (Fig. 4). The radiolucent line around the greater trochanter was first observed at postoperative 1 year. However, the line did not progress distally. This patient was followed-up for 2.6 years and did not complain about discomfort or pain around the hip joint or thigh. However, long-term follow-up should be necessary to evaluate the progression of loosening of the stem in this patient. In the present study, even though short proximal wedged stems were used for femoral neck fractures with osteoporosis, morphological mismatch of the proximal femur and the shape of the stem could occur. During preoperative planning for arthroplasty with a proximal wedged stem, care should be taken to avoid such morphological mismatch, even in cases of osteoporosis.

Thigh pain is one of the main concerns after hip replacement ${ }^{20)}$ and can be caused by various factors ${ }^{20,21)}$. Diaphyseal fitting and resulting stress shielding were considered as a common reason for thigh pain ${ }^{8}$. Theoretically, the use of a short stem might decrease thigh pain ${ }^{11}$. However, there is a controversy in this respect ${ }^{11,20)}$. Albers et al. ${ }^{11)}$ evaluated the mid-term results after THA with a Tri-lock BPS stem and reported a low rate $(1.6 \%)$ of postoperative thigh pain. However,
Amendola et al..$^{20)}$ evaluated the short- to mid-term results after THA with a short stem and reported a relatively high rate $(25 \%)$ of thigh pain after THA. The authors suggested that modulus mismatch between the stem tip and bone at the high stress subtrochanteric region might cause thigh pain. They also observed that the severity of thigh pain decreased with age. In the current study, we observed no thigh pain after hip arthroplasty in elderly patients with femoral neck fracture ${ }^{20}$. Less physical activity in older patients' age might cause less stress on the implant and reduce thigh pain in comparison with young active patients. Further study with a large number of cases is necessary to confirm the low incidence of thigh pain after hip arthroplasty in elderly patients.

The present study has a few limitations. First, it was a retrospective study with a relatively small number of patients is and is relatively short follow-up period. To support findings of the present study, a prospective study with more cases and longer follow-up will be necessary. Second, although patients without osteoporosis were excluded from the study, there were more Dorr type B hips than Dorr type $\mathrm{C}$ hips in the preoperative radiographs of the proximal femur. Further study with more Dorr type C femur cases will be necessary to confirm our results for this type of stem. Third, though the functional recovery of the patients was favorable in the current study, the result should be interpreted with caution. In the current study, the follow-up rate was relatively low. We only included patients who visited the clinic. Hence there would be a potential selection bias. Also, there are numerous factors which might affect the functional recovery including cognitive status, nutritional status or type of operation ${ }^{22,23)}$, but we did not evaluate the factors. Further study might be necessary to confirm the clinical finding of the current study.

\section{CONCLUSION}

The present study has documented favorable shortterm functional results of arthroplasty with a cementless proximal wedged short stem for femoral neck fracture with osteoporosis. No thigh pain, loosening, or osteolysis were observed, and bone ingrowth was stable in most cases, even with short-term follow-up. The short metaphyseal-stabilizing tapered stem could be a reliable treatment option for osteoporotic femoral neck fracture. Further study with longer follow-up would be necessary to support findings of the present study. 


\section{CONFLICT OF INTEREST}

The authors declare that there is no potential conflict of interest relevant to this article.

\section{REFERENCES}

1.Cicek H, Seyfettinoglu F, Kilicarslan K, Ogur HU, Öztürk $\mathrm{L}$, İnkaya E. What should be the preferred choice of hemiarthroplasty technique in American Society of Anesthesiologists (ASA) class III patients with femoral neck fractures? Cemented or cementless. Injury. 2015; 46:1567-70.

2.Lee YK, Kim KC, Yoon BH, Ha YC, Koo KH. Current trends of stem use in hemiarthroplasty for femoral neck fracture in South Korea. Clin Orthop Surg. 2014;6:285-9.

3. Hossain M, Andrew JG. Is there a difference in perioperative mortality between cemented and uncemented implants in hip fracture surgery? Injury. 2012;43:2161-4.

4.Lee YK, Joung HY, Kim SH, Ha YC, Koo KH. Cementless bipolar hemiarthroplasty using a micro-arc oxidation coated stem in patients with displaced femoral neck fractures. J Arthroplasty. 2014;29:2388-92.

5. Taylor F, Wright M, Zhu M. Hemiarthroplasty of the hip with and without cement: a randomized clinical trial. $J$ Bone Joint Surg Am. 2012;94:577-83.

6. Bonutti PM, Stroh AD, Issa K, Harwin SF, Patel DV, Mont MA. Proximally coated cementless bipolar hemiarthroplasty in Dorr type C bone. Orthopedics. 2014;37:e345-50.

7. Kim $\mathrm{YH}, \mathrm{Oh} \mathrm{JH}$. A comparison of a conventional versus a short, anatomical metaphyseal-fitting cementless femoral stem in the treatment of patients with a fracture of the femoral neck. J Bone Joint Surg Br. 2012;94:774-81.

8. Khanuja HS, Vakil JJ, Goddard MS, Mont MA. Cementless femoral fixation in total hip arthroplasty. $J$ Bone Joint Surg Am. 2011;93:500-9.

9. Vidovic D, Matejcic A, Punda M, et al. Periprosthetic bone loss following hemiarthroplasty: a comparison between cemented and cementless hip prosthesis. Injury. 2013;44 Suppl 3:S62-6.

10. Khanuja HS, Banerjee S, Jain D, Pivec R, Mont MA. Short bone-conserving stems in cementless hip arthroplasty. J Bone Joint Surg Am. 2014;96:1742-52.

11. Albers A, Aoude AA, Zukor DJ, Huk OL, Antoniou J, Tanzer M. Favorable results of a short, tapered, highly porous, proximally coated cementless femoral stem at a minimum 4-year follow-up. J Arthroplasty. 2016;31:824-9.

12. Garden RS. Low-angle fixation in fractures of the femoral neck. J Bone Joint Surg Br. 1961;43:647-63.

13. Dorr LD, Faugere MC, Mackel AM, Gruen TA, Bognar B, Malluche HH. Structural and cellular assessment of bone quality of proximal femur. Bone. 1993;14:231-42.

14. Koval KJ, Aharonoff GB, Rosenberg AD, Bernstein RL, Zuckerman JD. Functional outcome after hip fracture. Effect of general versus regional anesthesia. Clin Orthop Relat Res. 1998;(348):37-41.

15. Joshi RP, Eftekhar NS, McMahon DJ, Nercessian OA. Osteolysis after Charnley primary low-friction arthroplasty. A comparison of two matched paired groups. J Bone Joint Surg Br. 1998;80:585-90.

16. Martell JM, Pierson RH 3rd, Jacobs JJ, Rosenberg AG, Maley M, Galante JO. Primary total hip reconstruction with a titanium fiber-coated prosthesis inserted without cement. J Bone Joint Surg Am. 1993;75:554-71.

17. Loudon JR, Charnley J. Subsidence of the femoral prosthesis in total hip replacement in relation to the design of the stem. J Bone Joint Surg Br. 1980;62-B:450-3.

18. Engh CA, Bobyn JD, Glassman AH. Porous-coated hip replacement. The factors governing bone ingrowth, stress shielding, and clinical results. J Bone Joint Surg Br. 1987;69:45-55.

19. Rhyu KH, Lee SM, Chun YS, Kim KI, Cho YJ, Yoo MC. Does osteoporosis increase early subsidence of cementless double-tapered femoral stem in hip arthroplasty? J Arthroplasty. 2012;27:1305-9.

20. Amendola RL, Goetz DD, Liu SS, Callaghan JJ. Two- to 4year followup of a short stem THA construct: excellent fixation, thigh pain a concern. Clin Orthop Relat Res. 2017;475:375-83.

21. Baert IAC, Lluch E, Van Glabbeek F, et al. Short stem total hip arthroplasty: potential explanations for persistent post-surgical thigh pain. Med Hypotheses. 2017;107:4550.

22. Pareja Sierra T, Bartolomé Martín I, Rodríguez Solís J, et al. Predictive factors of hospital stay, mortality and functional recovery after surgery for hip fracture in elderly patients. Rev Esp Cir Ortop Traumatol. 2017;61:427-35.

23. Iaboni A, Rawson K, Burkett C, Lenze EJ, Flint AJ. Potentially inappropriate medications and the time to full functional recovery after hip fracture. Drugs Aging. 2017;34:723-8. 